\title{
Use of narrow band imaging in a group endoscopy practice
}

Author

Institution

\section{Patrick Yachimski}

Division of Gastroenterology, Hepatology and Nutrition, Vanderbilt University Medical Center, Nashville, Tennessee, United States received: 6. November 2013 accepted after revision: 9. December 2013

\section{Bibliography}

Dol http://dx.doi.org/

10.1055/s-0033-1359256

Endoscopy International Open

2013; 1: E8-E11

(c) Georg Thieme Verlag KG

Stuttgart · New York

E-ISSN 2196-9736

Corresponding author:

Patrick Yachimski, MD, MPH,

Division of Gastroenterology, Hepatology \& Nutrition,

Vanderbilt University Medical Center, 1660 The Vanderbilt Clinic,

Nashville, TN 37232-5280,

United States

patrick.yachimski@vanderbilt. edu

\section{License terms}

Background and study aim: Narrowband imaging (NBI) is an enhanced endoscopic optical technique which filters white light and accentuates imaging of the mucosal vasculature. Potential clinical applications of NBI include endoscopic inspection of Barrett's esophagus and endoscopic diagnosis of colorectal polyps. The degree to which NBI use has been adopted into clinical practice is unknown. The study objective was to identify the rate of NBI use in patients undergoing elective esophagoduodenoscopy (EGD) and colonoscopy, and to identify procedural factors associated with NBI use.

Methods: Elective endoscopic procedures were prospectively observed over a 2-week study period. NBI use during diagnostic EGD or colonoscopy was recorded in blinded fashion.

Results: NBI use was observed in $6.6 \%$ (21/318) of procedures, including $4.7 \%$ (5/106) of EGDs and

\section{Introduction}

The growth of diagnostic endoscopy has been facilitated by numerous advances in imaging technology. These include evolution from fiberoptic imaging to generation of images using a chargecoupled device and high definition digital imaging. Enhanced endoluminal imaging techniques have included chromoendoscopy and modalities that aspire to optical biopsy.

Among techniques for enhanced optical diagnosis, narrow band imaging (NBI) is a proprietary imaging modality in which the endoscope processor filters standard white light to specific wavelengths in the blue - green spectrum $(415 \mathrm{~nm}$ and $540 \mathrm{~nm}$ ). NBI thereby capitalizes on the peak absorption of hemoglobin and has the ability to accentuate visualization of the mucosal vasculature [1]. Proposed clinical applications of NBI include endoscopic evaluation of Barrett's esophagus and endoscopic diagnosis of colorectal polyps. Studies of NBI in endoscopic inspection of Barrett's
$7.5 \%(16 / 212)$ of colonoscopies. There was no difference in rate of NBI use when comparing EGD with or without biopsy, or when comparing colonoscopy with or without biopsy. NBI use was significantly higher in colonoscopy with polypectomy compared with colonoscopy without polypectomy ( $13 \%$ [10/77] vs. $4.4 \%$ [6/135], $P=0.03$ ). NBI use varied significantly among endoscopists. There was no association between patient type (outpatient vs. inpatient), procedure start time, sedation type, or trainee involvement and use/ non-use of NBI. Procedural documentation of NBI use was limited.

Conclusions NBI use was observed in $6.6 \%$ of elective endoscopic procedures and was highest in colonoscopies with polypectomy. Rate of NBI use varied significantly among endoscopists. Additional studies are needed to assess the magnitude of impact of NBI on routine endoscopic practice.

esophagus have demonstrated high sensitivity of NBI in detection of Barrett-associated high grade dysplasia [2], and the ability of NBI to detect dysplasia in a higher proportion of patients with fewer biopsy samples compared with standard white light endoscopy [3]. With respect to colorectal polyps, an NBI-based classification scheme has been developed which may accurately distinguish adenomatous from hyperplastic polyps [4], although it is not certain that use of NBI improves polyp detection rates [5-7].

Despite data regarding potential gastrointestinal endoscopic applications of NBI, the degree to which NBI use has been adopted into clinical practice is unknown. As with any new medical technology, legitimate questions exist regarding the degree to which efficacy of NBI as demonstrated in clinical studies will translate into effective use of NBI in actual practice [8]. The aim of this study, therefore, was to prospectively define the rate of NBI use among patients referred to a large group endoscopy practice for diagnostic endoscopy 
(esophagogastroduodenoscopy and colonoscopy), and to identify procedural factors associated with NBI use.

\section{Methods}

Approval to conduct this study as a quality assurance protocol was granted by the Institutional Review Board at the study institution.

This study was conducted at the endoscopy center of a tertiary care academic center, where both inpatient and outpatient procedures are performed in a hospital-based suite. Each procedure room is equipped with NBI capability (180 series gastroscope or colonoscope, CV-180 Evis Exera II video processor, and CLV-180 light source; Olympus Medical Systems, Tokyo, Japan).

Elective diagnostic procedures were prospectively observed over a 2-week period. Each consecutive esophagogastroduodenoscopy (EGD) and colonoscopy was directly observed by in-room endoscopy technicians, who assist the endoscopist with equipment setup and when endoscopic accessory use (i.e. biopsy forceps, polypectomy snare, endoscopic hemostatic device, etc.) is required. A technician is present in each procedure room for the entire duration of each procedure. For each eligible procedure, the technician documented whether or not NBI was used. Faculty endoscopists observed during this time period were unaware of the study. Procedures performed by the study author were not included; otherwise all faculty endoscopists were eligible for observation.

The following endoscopic procedures were excluded from the analysis: EGD or colonoscopy with therapeutic intent, specifically endoscopic dilation, endoluminal stent maneuvers, or delivery of endoscopic hemostatic therapy; EGD for nasoenteral or percutaneous feeding tube placement; balloon- or spiral-assisted smallbowel enteroscopy; endoscopic retrograde cholangiopancreatography; EGD with endoscopic ultrasound; ileoscopy and pouchoscopy.

Following completion of the 2-week observational study period, operative reports including reports generated by Olympus Endoworks software and dictated operative notes were manually reviewed by the study author, who was not blinded to study design or intent. The following data were extracted: procedure type, procedure indication, identification of attending endoscopist, trainee involvement, patient type (inpatient versus outpatient), procedure start time (a.m. vs. p.m.), sedation-type (endoscopistdirected conscious or deep sedation vs. monitored anesthesia care with anesthesia staff support); performance of endoscopic polypectomy, either by snare or forceps biopsy; performance of endoscopic tissue biopsy for intent other than polypectomy.

Extracted data were stored in a Microsoft Excel spreadsheet, and statistical analyses were performed using JMP 10.0.0 software (SAS Institute, Cary, NC, USA). The chi-squared or Fisher's exact test was used for comparison of categorical variables. Two-sided $P$ values of $<0.05$ were considered statistically significant.

\section{Results}

$\nabla$

During the two-week observational study period in July-August 2013, data regarding NBI use were recorded for 318 elective endoscopic procedures, consisting of 106 EGDs and 212 colonoscopies. Additional procedural data are summarized in $\bullet$ Table 1.
Table 1 Procedure data for 318 elective endoscopic procedures in a large group endoscopy practice

\begin{tabular}{|l|l|}
\hline Procedure type & $\mathbf{n}(\%)$ \\
\hline EGD & $106(33)$ \\
\hline $\begin{array}{l}\text { Colonoscopy } \\
\text { Sedation type }\end{array}$ & $212(67)$ \\
\hline Conscious sedation & $197(62)$ \\
\hline MAC & $121(38)$ \\
\hline Patient type & $305(96)$ \\
\hline $\begin{array}{l}\text { Outpatient } \\
\text { Inpatient }\end{array}$ & $13(4)$ \\
\hline Procedure start time & $195(61)$ \\
\hline a.m. & $123(39)$ \\
\hline p.m. & $14(4)$ \\
\hline Trainee involvement \\
\hline Yes \\
\hline No
\end{tabular}

Data presented as N (\%).

EGD, esophagogastroduodenoscopy; MAC, monitored anesthesia care.

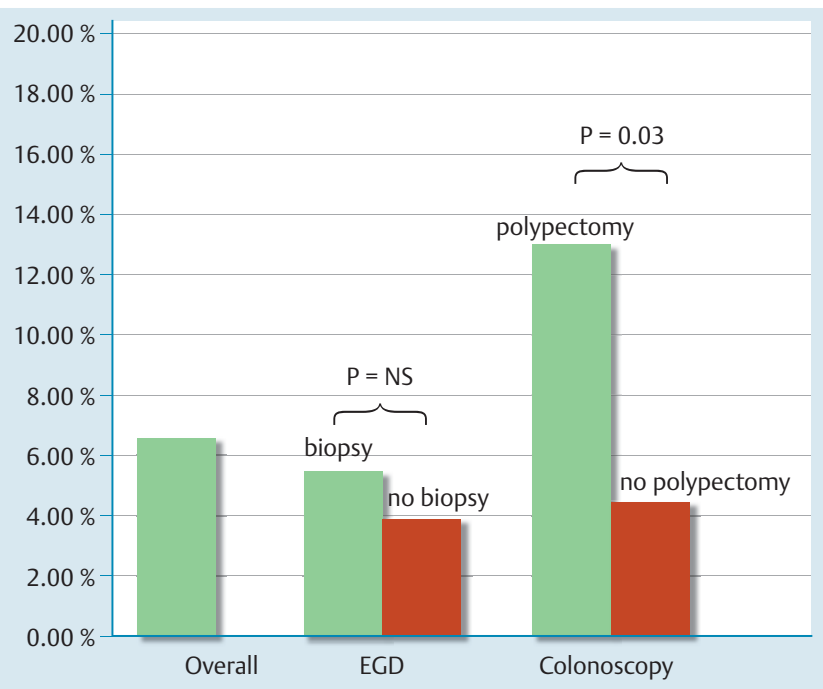

Fig. 1 Rates of narrow band imaging (NBI) use for all study procedures, esophagoduodenoscopy (EGD) with and without biopsy, colonoscopy with and without polypectomy. NS, not significant.

The most common indications for EGD were evaluation of abdominal pain/dyspepsia (22\%) and evaluation of gastroesophageal reflux disease (19\%). Additional indications included evaluation of weight loss, anemia or suspected gastrointestinal bleeding, and diarrhea. The most common indication for colonoscopy was screening/surveillance for colorectal cancer (56\%). Additional indications included evaluation of suspected or established inflammatory bowel disease, anemia or suspected gastrointestinal bleeding, and diarrhea.

NBI use was observed in $6.6 \%(21 / 318)$ of procedures. This included use of NBI in $4.7 \%$ (5/106) of EGDs and 7.5\% (16/212) of colonoscopies ( $P=0.47$ for comparison). No difference in rate of NBI use was found when comparing EGD with or without biopsy (5.5\% [3/55] vs. 3.9\% [2/51]; $P=1$ ), or when comparing colonoscopy with or without biopsy $(10.5 \%$ [8/76] vs. $5.9 \%$ [8/136]; $P=$ $0.28)$. NBI use was significantly higher in colonoscopy with polypectomy when compared with colonoscopy without polypectomy (13\% [10/77] vs. $4.4 \%$ [6/135]; $P=0.03$ ) ( $\bullet$ Fig. 1 ). 
On univariate analysis, there was no association between patient type (outpatient vs. inpatient), procedure start time, sedation type, or trainee involvement and use/non-use of NBI. For both EGD and colonoscopy, there was no association between procedure indication and use/non-use of NBI. NBI use was observed in zero of seven EGDs with a documented primary procedural indication of screening for or surveillance of Barrett's esophagus. NBI use was observed in 2 of 9 (22\%) colonoscopies with a documented primary indication of surveillance in the setting of chronic ulcerative colitis. No use of an alternative imagine modality, such as methylene blue chromoendoscopy or high magnification endoscopy was described in any operative report.

Observed endoscopic procedures were performed by 23 faculty endoscopists, with a procedure volume ranging from 1 to 58 per endoscopist during the 2-week study period. The rate of NBI use ranged from 0 to $100 \%$ of procedures per endoscopist. After excludsion of endoscopists who performed fewer than five endoscopies during the study period,the rate of NBI use for the remaining 15 endoscopists ranged from 0 to $23 \%$. There was a significant difference in rate of NBI use $(P<0.01$ for overall comparison) amongst these 15 endoscopists. NBI use among the 5 highest volume endoscopists ranged from 0 to $16 \%$.

Among cases with observed NBI use, photodocumentation of NBI use was present in $24 \%$ (5/21) of operative reports. There was no text or written documentation of NBI use in any operative report.

\section{Discussion}

$\nabla$

This observational study detected use of NBI in $6.6 \%$ of elective endoscopic procedures. The highest rate of NBI use was observed in colonoscopies with polypectomy (13\%), and this rate was significantly higher than that observed in colonoscopies without polypectomy. This study also detected a significant difference in the rate of NBI use in global comparison of individual endoscopists. Specific factors that influence the adoption of NBI use into routine practice by individual endoscopists were not examined in this study.

Endoscopists were not asked to self-report NBI use and were not informed of the study. The study was designed in this fashion to avoid the potential of a Hawthorne effect, wherein performance may be influenced in subjects who are aware that they are being observed. The endoscopists were blinded to the study, making the study design and findings unique. Published data regarding rate of NBI use in actual practice are virtually nonexistent. A survey of European university hospitals reported use of NBI or alternative commercial enhanced-imaging technology in $67 \%$ of institutions in the evaluation of Barrett's neoplasia [9], but did not report a per-case use rate.

In the current study, there was no a priori hypothesis regarding the rate of NBI use expected in this observational study, as there are limited standard recommendations or guidelines for routine NBI use in diagnostic endoscopy, and existing guidelines are open to flexible interpretation. For instance, the American Gastroenterological Association medical position statement on Barrett's esophagus suggests that chromoendoscopy or electronic chromoendoscopy is not necessary in the routine endoscopic surveillance of Barrett's esophagus, but may be helpful in guiding biopsies for patients with dysplasia or visible mucosal abnormalities [10]. The rate of NBI use in this study can therefore not be subjectively designated as high or low, but instead serves as a baseline metric in this study setting against which future NBI use can be measured.

Adoption of NBI use in routine diagnostic endoscopy may have implications for application of future enhanced-imaging technologies. In the hands of the endoscopist, NBI is a fast and efficient enhanced-imaging technology. No additional equipment is required other than an existing video monitor and NBI-equipped scope and processor. An endoscopist can toggle between NBI and standard white light literally in seconds, without use of additional endoscopic devices, accessories, or medications. And while there may be a learning curve for interpretation of NBI images $[11,12]$, endoscopist interpretation of endoluminal NBI images would seem less a departure from white light endoluminal images than interpretation of images generated by other optical techniques, such as endomicroscopy, that focus on cellular structures. Based on these criteria, one would speculate that adoption and use of imaging technologies requiring additional equipment or capital investment, additional time with respect to procedure duration, and/or increasingly complex image analysis would be lower than that for NBI.

Finally, it is worthwhile to note that documentation of NBI use was uncommon. Photodocumentation of NBI use was provided in $24 \%$ (5/21) of operative reports from cases in which NBI use was observed. There was no text or written documentation of NBI use in any operative report. Future consideration may be warranted as to whether documentation guidelines should exist for procedural use of NBI or other adjunct imaging modalities. An American Society of Gastrointestinal Endoscopy Preservation and Incorporation of Valuable endoscopic Innovations (PIVI) statement on real-time endoscopic assessment of histology of colon polyps suggests that lesion photocumentation is necessary if a resect-and-discard strategy is to be implemented [13].

While extending the study duration to increase sample size would increase statistical power and eliminate the possibility of type II error in examining factors associated with NBI use, the study as completed is likely to have strong internal validity, in that the observed range of endoscopic procedures during the 2week time period is likely to offer an accurate representation of the range of endoscopic practice in this setting. It may not be possible, however, to generalize the study findings to other institutions or endoscopy settings which may have differing practice patterns.

Limitations of the current study include the potential for misclassification of NBI use. Instances of NBI use may not have been documented if not observed or not recognized by the endoscopy technician. An alternative would have been to video record the entire procedure for subsequent review; however maintenance of adequate blinding with this approach would be challenging. Documented cases of NBI use cannot distinguish between intentional and unintentional NBI use-for instance, if the endoscopist had inadvertently pressed the button on the scope handle for application of NBI when he/she had intended instead to press the button for image capture. In addition, there was limited heterogeneity in some of the procedural variables (e.g. trainee involvement) to adequately assess for potential association with NBI use/ non-use. A post hoc survey of participating endoscopists might offer insight as to whether their opinions on the role of NBI match their actual practice, but has no bearing on the aim of this study, which was to objectively document rate of NBI use.

In summary, NBI use was observed in $6.6 \%$ of elective endoscopic procedures. Use of NBI was highest in colonoscopies with polypectomy. Rate of NBI use varied significantly among endosco- 
pists. Additional large-scale prospective data are needed to assess the magnitude of impact of NBI on routine endoscopic practice.

\section{Competing interests: None.}

\section{Acknowledgement}

$\nabla$

The author wishes to acknowledge the help and efforts of the Vanderbilt University GI Endoscopy Lab nursing and technical staff in completion of this study.

\section{References}

1 Gono K, Obi T, Yamaguchi M et al. Appearance of enhanced tissue features in narrow-band endoscopic imaging. J Biomed Opt 2004; 9: 568-577 PubMed

2 Mannath J, Subramanian V, Hawkey CJ et al. Narrow band imaging for characterization of high grade dysplasia and specialized intestinal metaplasia in Barrett's esophagus: a meta-analysis. Endoscopy 2010; 42: 351-359 PubMed

3 Wolfsen HC, Crook JE, Krishna M et al. Prospective, controlled tandem endoscopy study of narrow band imaging for dysplasia detection in Barrett's Esophagus. Gastroenterology 2008; 135: 24-31 PubMed

4 Hewett DG, Kaltenbach T, Sano Y et al. Validation of a simple classification system for endoscopic diagnosis of small colorectal polyps using narrow-band imaging. Gastroenterology 2012; 143: 599-607 e1 PubMed

5 Nagorni A, Bjelakovic G, Petrovic B. Narrow band imaging versus conventional white light colonoscopy for the detection of colorectal polyps. Cochrane Database Syst Rev 2012; 1: CD008361 10.1002/ 14651858. CD008361. pub2 PubMed

6 Pasha SF, Leighton JA, Das A et al. Comparison of the yield and miss rate of narrow band imaging and white light endoscopy in patients undergoing screening or surveillance colonoscopy: a meta-analysis. Am J Gastroenterol 2012; 107: 363 - 370 PubMed quiz 371

7 Rastogi A, Early DS, Gupta $N$ et al. Randomized, controlled trial of standard-definition white-light, high-definition white-light, and narrow-band imaging colonoscopy for the detection of colon polyps and prediction of polyp histology. Gastrointest Endosc 2011; 74: $593-$ 602 PubMed

8 Cash BD. Narrow-band imaging for colorectal polyps: it can be taught but will it be used? Gastrointest Endosc 2010; 72: 577-579 PubMed

9 Heuberger $D$, Manner $H$, Ell $C$ et al. How is early Barrett's cancer currently diagnosed and treated in Western Europe? Results of a survey at 52 university hospitals in eight Western European countries Z Gastroenterol 2012; 50: 670-676 PubMed

10 Spechler SJ, Sharma P, Souza RF et al. American Gastroenterological Association medical position statement on the management of Barrett's esophagus. Gastroenterology 2011; 140: 1084 - 1091 PubMed

11 Dai J, Shen YF, Sano Y et al. Evaluation of narrow-band imaging in the diagnosis of colorectal lesions: is a learning curve involved? Dig Endosc 2013; 25: 180-188 PubMed

12 Patel SG, Rastogi A, Austin G et al. Gastroenterology trainees can easily learn histologic characterization of diminutive colorectal polyps with narrow band imaging. Clin Gastroenterol Hepatol 2013; 11: $997-$ 1003 e1 PubMed

13 Rex DK, Kahi C, O'Brien M et al. The American Society for Gastrointestinal Endoscopy PIVI (Preservation and Incorporation of Valuable Endoscopic Innovations) on real-time endoscopic assessment of the histology of diminutive colorectal polyps. Gastrointest Endosc 2011; 73: 419-422 PubMed 